\title{
TTR
}

Traduction, terminologie, rédaction

\section{Eva C. Karpinski. Borrowed Tongues: Life Writing, Migration, and Translation. Waterloo, Ontario, Wilfrid Laurier University Press, 2012, 271 p.}

\section{Aurelia Klimkiewicz}

Volume 27, numéro 1, 1er semestre 2014

URI : https://id.erudit.org/iderudit/1037126ar

DOI : https://doi.org/10.7202/1037126ar

Aller au sommaire du numéro

Éditeur(s)

Association canadienne de traductologie

ISSN

0835-8443 (imprimé)

1708-2188 (numérique)

Découvrir la revue

Citer ce compte rendu

Klimkiewicz, A. (2014). Compte rendu de [Eva C. Karpinski. Borrowed Tongues: Life Writing, Migration, and Translation. Waterloo, Ontario, Wilfrid Laurier University Press, 2012, 271 p.] TTR, 27(1), 234-239.

https://doi.org/10.7202/1037126ar d'utilisation que vous pouvez consulter en ligne.

https://apropos.erudit.org/fr/usagers/politique-dutilisation/ 
Chevrel, Yves (1995). «La réception des littératures étrangères ». Revista de Filologia Francesa, 7, p. 83-100.

Marie-France Guénette Université de Montréal

\section{Eva C. Karpinski. Borrowed Tongues: Life Writing, Migration, and Translation. Waterloo, Ontario, Wilfrid Laurier University Press, 2012, 271 p.}

Consacré à l'analyse des écrits autobiographiques des femmes migrantes, l'ouvrage d'Eva C. Karpinski pose le problème d'autoreprésentation qui se fait dans la langue de l'autre qu'elle appelle empruntée (borrowed). À cet égard, le choix terminologique n'est pas innocent, car il renvoie aux relations de pouvoir ancrées dans les inégalités historiques, économiques, raciales ou encore celles liées à l'identité sexuelle. En s'inscrivant dans le paradigme poststructuraliste qui prône l'inachèvement de la production $\mathrm{du}$ sens et en s'appuyant plus précisément sur les postulats déconstructivistes, féministes, postcoloniaux, culturalistes et traductologiques, l'auteure propose de cerner le rôle que la traduction assume dans la production des récits de vie relatant différentes expériences de migration, ainsi que d'identifier les stratégies d'écriture qui font appel aux procédés de transfert alors que la mise en récit de soi se fait dans la langue empruntée.

Clairement interdisciplinaire, la démarche de Karpinski permet de dévoiler les mécanismes de la construction identitaire (approche culturaliste) et, par conséquent, d'aborder la traduction en déjouant l'opposition entre le soi et l'autre, opposition forçant à maintenir la frontière qui, par le fait même, facilite l'exclusion ou l'enfermement de l'autre dans un lieu figé (approche féministe). Examiner les questions pour lesquelles on s'aligne sur telle ou telle identité donne par contre la possibilité de scruter la traduction en tant que partie intégrante de la formation des représentations et des échanges culturels (approche postcoloniale).

Dans son analyse, l'auteure aborde donc la traduction à partir de la position épistémologique décentrée en empruntant à Derrida son concept de «supplément» pour parler de la femme migrante qui doit forger son propre lieu énonciatif en fonction de la situation linguistique, culturelle, identitaire ou existentielle 
au nom de laquelle elle s'exprime en tant que femme survivante, colonisée, non occidentale, non blanche, etc., aux prises avec l'absence d'un genre discursif ou textuel qui rendrait possible la mise en récit de son expérience. Contrairement à l'autobiographie qui est un produit de la société patriarcale, le récit de vie s'impose justement comme forme d'écriture libre et malléable, ayant la capacité d'accueillir la diversité des expériences de celles qui sont exclues de l'histoire officielle.

À la différence de l'assimilation dont l'objectif est d'effacer la différence, le concept de positionnement sert à témoigner des tensions identitaires et des problèmes d'appartenance. C'est pourquoi au cœur de l'analyse de Karpinski se trouvent des éléments qui récusent la traduction transparente, car dans le contexte de migration, l'emprunt, l'intraduisible et l'inassimilable résistent à la circulation fluide du sens (du locuteur vers l'interlocuteur, de l'original vers la copie traduite) en déployant un réseau de références plus complexes et plus chaotiques qui fait voir l'impossibilité de production, de reproduction et de divulgation du sens à partir d'un seul centre d'énonciation. Une vision du monde unique et monologique ne peut exister que lorsquelle se sert de la violence physique, économique ou symbolique. La traduction non transparente témoignerait donc de la résistance à gommer cette violence ainsi que de la nécessité de contester la redistribution des rôles selon les mêmes règles établies par le plus fort.

Dans ce contexte, comment situer la traduction alors que l'accent est mis sur la parole de la femme migrante qui doit forger son propre genre littéraire (le récit de vie au féminin), qui doit s'approprier une langue qui n'est pas la sienne (la langue patriarcale/ étrangère/coloniale) et qui est forcée de créer un lien social malgré les barrières linguistiques, culturelles et même existentielles dans le cas de celles qui ont survécu les traumatismes liés aux événements tels que la guerre, le génocide, le camp de réfugiés, le viol, le racisme ou l'exclusion? Tout d'abord, il est question d'inscrire la traduction dans un contexte atypique défini comme proximité physique et spatiale, mais dépourvue de véritable médiation étant donné que le lien social et humain a été perverti par la violence du colonialisme, du racisme, du patriarcat ou encore à cause de la vulnérabilité de la femme migrante dans la société d'accueil. Ensuite, le contexte de migration invite à insérer la traduction dans une dynamique intergénérationnelle qui s'opère au moyen de l'activité intense et 
incessante de traduction, manifeste dans la volonté de transmettre l'héritage personnel, familial et culturel, ici entre la mère et la fille, entre le pays d'origine et la société d'accueil. Finalement, il s'agit de saisir le travail de traduction au niveau de l'identité aux prises avec la fragmentation et la stratification de la subjectivité mettant en scène les conflits internes quant à l'appartenance ethnique, religieuse, raciale et sexuelle.

En se penchant sur huit récits de vie publiés par les femmes migrantes en anglais au Canada et aux États-Unis entre les années dix et quatre-vingt-dix du $\mathrm{XX}^{\mathrm{e}}$ siècle, Karpinski souligne le défi que posent l'écriture et l'(auto)traduction à partir de la position du sujet migrant et hybride alors que celui-ci s'adresse à un public récepteur plutôt monolingue et homogène et cela dans une langue dominante incarnant la suprématie occidentale, blanche et patriarcale. En guise de réponse, dans les textes analysés, on trouve différentes stratégies d'écriture à l'œuvre permettant de suivre les changements de positionnement vis-à-vis le processus d'immigration, la société d'accueil, l'héritage d'origine, le problème de transmission, le résidu mémoriel intraduisible, ainsi de suite.

Dans le premier chapitre intitulé «Literacy Narratives: Mary Antin and Laura Goodman Salverson", il est question d'analyser deux récits de vie du début du $\mathrm{XX}^{\mathrm{e}}$ siècle: The Promised Land: The Autobiography of a Russian Immigrant (1912) de Mary Antin qui raconte sa propre histoire d'immigration et Confessions of an Immigrant's Daughter (1939) de Laura Goodman Salverson qui, quant à elle, parle au nom de la deuxième génération d'immigrants. Bien que les deux textes s'inscrivent à première vue dans le discours triomphaliste de l'assimilation si caractéristique de cette époque, leur analyse plus détaillée permet de voir que tout en voulant respecter les attentes des politiques éditoriales et des lecteurs natifs du pays d'accueil, les deux auteures se servent des stratégies qui témoignent plutôt de l'ambivalence au sujet de la négociation identitaire entre l'assimilation, la résistance et la subversion. L'acte d'écrire signifie aussi la prise de conscience du rôle que joue la traduction non seulement dans le processus d'adaptation, mais aussi dans l'apprentissage actif de la langue étrangère, sans parler de la possibilité de transmettre la mémoire culturelle.

Le deuxième chapitre, "Immigrant Crypto(auto)graphy: Akemi Kikumura and Apolonja Maria Kojder», pose le problème de la généalogie matrilinéaire au sein de laquelle la fille assume 
le rôle de médiateur culturel (cultural broker) responsable de transmettre l'histoire orale de l'immigration familiale qui fait partie, quant à elle, d'une histoire plus vaste, celle du multiculturalisme. Comment donc traduire - au sens de transmettre - du privé vers l'officiel, d'une langue faible vers une langue dominante, de l'oral vers lécrit, de la marge vers le centre, du silence vers l'audible, du chaotique vers l'articulé? En même temps, comment rester fidèle au récit maternel sans l'assimiler en son for intérieur, mais lui trouver une forme de transmission au-delà de la dispersion? Comme le montre l'analyse des livres d'Akemi Kikumura, Through Harsh Winters: The Life of a Japanese Immigrant Woman (1981), et d'Apolonja Maria Kojder, Marynia, Don't Cry: Memoirs of Two Polish-Canadian Families (1995), la tâche n'est pas facile, même quand l'effort de traduction/transmission s'appuie sur un travail de réflexion théorique aboutissant à employer différentes formes de médiation, que ce soit au niveau de la transcription, des stratégies éditoriales ou de l'approche translative. Dans ce chapitre en particulier, Karpinski expose le danger qui guette le traitement du récit de vie comme auto-ethnographie une fois que le témoignage oral (ici de la mère) tombe entre les mains du spécialiste (ici la fille) qui procède à l'effacement de la différence en évacuant l'accent, l'hétérogénéité et l'oralité par respect à l'anglais standard et aux politiques éditoriales souvent avides d'histoires d'immigration réussies. Pour que la traduction incarne véritablement le passage intergénérationnel qui garantirait la survie de la mémoire, il faudrait poser le problème de la traduction non pas en fonction des attentes du public récepteur, mais le faire, à la manière de Benjamin et Derrida, dans une dynamique de don et de dette, autrement dit comme réponse responsable à l'appel de témoigner.

Dans le troisième chapitre intitulé «Experimental SelfTranslations: Eva Hoffman and Smaro Kamboureli», Karpinski place la traduction sous le sceau de la négativité parce quelle manque à son devoir de transmission surtout à cause de son incapacité d'échapper à la logique de la perte. Les deux récits autoréflexifs discutés dans ce chapitre, Lost in Translation d'Eva Hoffman (1989) et In the Second Person de Smaro Kamboureli (1985), dévoilent les embûches du processus de construction de la subjectivité dans un contexte d'immigration d'emblée éclaté. Si Hoffman exprime le penchant pour l'affaiblissement des liens avec le passé au nom de l'assimilation allant jusqu'à l'effacement 
de l'accent et la transformation du langage corporel, Kamboureli valorise la non-traduction et signale que la migration offre le privilège épistémologique de voir à partir des perspectives diversifiées. Par contre, toutes les deux performent la traduction dans leur constante négociation sur le plan de la langue et de la subjectivité. De même, elles sont conscientes que la standardisation de la langue est un instrument de contrôle social et d'exclusion des non-natifs.

Mais c'est le dernier chapitre du livre, intitulé «Translation as Allegorical Metafiction: Marlene Nourbese Philip and Jamaica Kincaid », qui situe la traduction dans un contexte des plus radicaux, car il s'agit des enjeux postcoloniaux allant du dépouillement de l'héritage culturel jusqu'au racisme et à l'exclusion de l'histoire. Les tentatives d'introduction d'autres mémoires dans l'histoire officielle du pays d'accueil - discutées dans les chapitres précédents - cèdent ici la place à la production de la contre-mémoire. Les deux auteures "perform the work of translation as creative transformation and resistance to being fixed in the position of the 'other'» (p. 220). Dans Looking for Livingstone: An Odyssey of Silence (1991), Philip emploie, par exemple, différentes formes de traduction: transfert interlinguistique, traduction culturelle, traduction comme analyse du discours et retraduction, formes qui tentent ensemble de créer une zone de passage et un langage sans résidus de l'oppression. Quant à The Autobiography of My Mother (1996), Jamaica Kincaid met de l'avant «the position of a heterogeneous subject within the dominant symbolic order, speaking from within the system, but through the logic of negativity and rejection» (p. 220). C'est la réponse à une relation dépourvue d'amour entre le colonisé et le colonisateur d'un côté et de l'autre, la dépossession laissée en héritage à la communauté diasporique. Encore une fois, la traduction s'avère être une stratégie de résistance contre l'effacement des traces de la différence.

Voici une brillante étude qui traite d'un seul coup l'écriture au féminin, la littérature migrante, la transmission de la mémoire culturelle, le déplacement, l'identité, et cela par le biais de la traduction, qui nest plus exclusivement synonyme de contact et d'enrichissement culturel, mais plutôt symptôme des malaises qui se manifestent dans différents parcours migratoires et au sein des sociétés dites globales prônant l'uniformisation. Identifiée à la résistance contre les forces hégémoniques, la traduction, en effet, 
permet de défendre les différences et de dénoncer les logiques de leur effacement. L'analyse de Karpinski fait en même temps ressortir l'apport de la traduction au renouvellement des formes narratives, à la critique littéraire et à l'élargissement des perspectives théoriques, sans oublier son pouvoir curatif, car la traduction facilite la compréhension des expériences migratoires. Il ne faut pas oublier non plus son pouvoir de contestation, que ce soit à l'égard de l'assimilation, du nationalisme ou de la diaspora dont le mythe de la mobilité est désormais au service du néolibéralisme. Quant à la traductologie, trois postulats avancés dans cette étude sont dignes d'intérêt: 1) la traduction n'est pas l'enrichissement ou un simple contact mais une dette;2) la traduction inscrite dans une injustice suit ses propres règles; 3 ) l'expérience de migration altère le rapport à la traduction. Ces trois postulats sont intimement liés à l'éthique.

Aurelia Klimkiewicz UNIVERSITÉ YorK

Frederica Scarpa. La traduction spécialisée: une approche professionnelle à l'enseignement de la traduction. Trad. et adapt. Marco A. Fiola. Ottawa, Presses de l'Université d'Ottawa, 2010, $451 \mathrm{p}$.

La traduction spécialisée: une approche professionnelle à l'enseignement de la traduction est un ouvrage doté d'une double visée qui répond à de réels besoins : établir un état des lieux de la traduction spécialisée - le terme s'entend ici dans un sens restreint, soit la traduction de textes technico-scientifiques (p.3) - et fournir aux professeurs un outil utile à l'enseignement de cette discipline. L'auteure, Frederica Scarpa, étant italienne - elle est professeure à l'Université de Trieste -, louvrage se concentre principalement sur les recherches de ses compatriotes et sur l'enseignement de la traduction dans le contexte européen. C'est ce qui a poussé le traducteur, Marco A. Fiola, professeur à l'Université Ryerson de Toronto, à l'adapter aux réalités canadiennes. Plus précisément, le traducteur a maintenu le contenu théorique, retranché «tout ce qui portait exclusivement sur les particularités de l'italien» (p. VII), ajouté des références à des sources documentaires qu'il a jugé pertinentes et complété la bibliographie. 